\title{
A NEW TECHNIQUE FOR THE DETECTION OF PERIODIC SIGNALS IN "COLOURED" POWER SPECTRA
}

\author{
G. L. Israel ${ }^{1}$ \\ International School for Advanced Studies (SISSA-ISAS), V. Beirut 2-4, I-34014 Trieste, Italy \\ Dipartimento di Fisica, Università di Roma "La Sapienza", P.le A. Moro 2, I-00185, Roma, Italy \\ e-mail: gianluca@vega.sissa.it \\ and \\ L. Stella ${ }^{1,2}$ \\ Osservatorio Astronomico di Brera, Via E. Bianchi 46, I-22055 Merate (Lc), Italy \\ e-mail: stella@antares.merate.mi.astro.it \\ Accepted for publication in The Astrophysical Journal
}

\begin{abstract}
The light curves from a variety of celestial objects display aperiodic variations, often giving rise to red-noise components in their power spectra. Searching for a narrow power spectrum peak resulting from a periodic modulation over the frequency range in which these "coloured" noise components are dominant has proven a very complex task. Commonly used methods rely upon spectral smoothing or incoherent summation of sample spectra in order to decrease the variance of the power estimates. The consequent reduction in frequency resolution causes also a reduction of sensitivity to periodic signals.

We develop here a technique aimed at detecting periodicities in the presence of "coloured" power spectrum components, while mantaining the highest Fourier frequency resolution. First we introduce a simple approximation to the statistical properties of the "coloured" power spectra from celestial objects, based on a few examples and the theory of linear processes. We then estimate the continuum components in the power spectrum through an ad hoc smoothing technique. This involves averaging the spectral estimates adjacent to each frequency over a suitably chosen interval, in order to follow steep red-noise features and produce estimates that are locally unaffected by the possible presence of a sharp peak. By dividing the sample spectrum by the smoothed one, a white-noise like spectrum is obtained,
\end{abstract}

\footnotetext{
${ }^{1}$ Affiliated to the International Center for Relativistic Astrophysics

${ }^{2}$ Now at the Astronomical Observatory of Rome, Via dell'Osservatorio 2, I-00040, Monteporzio Catone (Roma), Italy
} 
the approximate probability distribution of which is derived. A search for coherent pulsations is then carried out by looking for peaks in the divided spectrum, the chance probability of which is below a given detection threshold. If no significant peaks are found, an upper limit to the amplitude of a sinusoidal modulation is worked out for each searched frequency.

The technique is tested and its range of applicability determined through extensive numerical simulations. We present also an application to the X-ray light curves of V0332+53, a highly variable accreting X-ray pulsar, and GX13+1, a bright and variable accreting source in the galactic bulge.

Subject headings: Numerical methods - Pulsation — Stars: Neutron — Pulsars — Oscillations - X-ray: Binaries 


\section{INTRODUCTION}

Since the prehistorical efforts aimed at developing the calendar, the detection and investigation of periodic phenomena has played a major role in astronomy. Crucial information is obtained through the observation and measurement of periodicities in many classes of celestial bodies encompassing all scales from comets and asteroids to the largest structures currently known at cosmological distances. In some cases periodic signals from the cosmos can be measured to an exceptionally high accuracy, that rivals that of atomic clocks (Kaspi, Taylor \& Ryba 1995). Astronomical time series of increased statistical quality, time resolution and duration have become available over the last two decades for a variety of objects and over different bands of the electromagnetic spectrum. Power spectrum analysis is probably the single most important technique that is applied to these series in order to: (a) detect periodicities (or quasi-periodicities) by the presence of significant power spetrum peaks; (b) characterise the noise variability through the study of continuum power spectrum components. In particular, recent applications to high energy astronomical time series have been especially numerous and successfull, as a consequence of the pronounced variability (often both periodic and aperiodic in nature) detected in many sources and the availability of long uninterrupted observations (up to several days) of high signal to noise ratio. The continuum power spectral components arising from noise variability usually increase towards lower frequencies (red noise), often in a power law-like fashion. Their study has proven to be a useful tool for morphological classifications and, sometimes, has provided constraints on physical models (e.g. Stella 1988; Hasinger \& van der Klis 1989; van der Klis 1995). The periodic modulations revealed in a number of high energy sources often arise from the rotation of compact magnetic stars, or the orbital motion of a binary system. The detection and accurate measurement of these periods provides a tool of paramount importance. A variety of other periodic or quasi-periodic phenomena in X-ray sources have been discovered over a range of timescales (from tens of milliseconds to years).

Astronomical observations rely more and more upon photon counting instruments; therefore, measurement errors are often dominated by the statistical uncertainties originating from the Poisson distribution of the counts. This translates into a white noise power spectrum component of known amplitude, which, after normalisation, follows a $\chi^{2}$ distribution with 2 degrees of freedom $\left(\chi_{2}^{2}\right)$. Any intrinsic variability of the source, either resulting from periodic signal(s) or from noise(s), must possess significant power above the counting statistics white noise component in order to be detected.

Traditionally, the detection of periodic signals through peaks in the sample spectrum has been carried out either (i) by eye, in all those cases in which the peak amplitude is so large that it is self-evident or (ii) by ruling out (at a given confidence level) that a peak originates from an underlying white noise. The latter technique implicitely assumes that the power spectra do not possess any conspicuous "coloured" component above the white noise. As mentioned above, this hypothesis is not verified at least over a range of frequencies in many instances. Indeed 
the very presence of "coloured" continuum power spectrum components resulting from source variability noise makes the detection of significant power spectrum peaks a difficult statistical problem. In general, establishing whether or not a sample spectrum peak originates from a periodic modulation requires an evaluation of the peak significance with respect to the local continuum of the sample spectrum which, in turn, can be dominated by the aperiodic variability of the source. Techniques along these lines have been developed, which often rely upon smoothing or incoherent summing of sample spectra to decrease the variance of the power estimates and/or allow the use of relatively simple statistics. In this way, however, the frequency resolution and, correspondingly, the sensitivity of the searches is reduced (e.g. Jenkins \& Watts 1968; van der Klis 1988). Moreover, standard spectral smoothing does not allow to reproduce power law-like spectral shapes with acceptable accuracy.

This paper describes a new technique for detecting power spectrum peaks arising from a periodic modulation, in the presence of "coloured" power spectrum components, while preserving the Fourier frequency resolution. In this technique, the continuum components of the spectrum at the $j$-th frequency are evaluated based on an ad hoc smoothing technique which involves averaging the spectral estimates adjacent to $j$-th frequency over a given logarithmic interval excluding the $j$-th frequency itself. The advantage of this type of smoothing is that, while it allows the continuum features of the power spectrum to be followed, it is locally unaffected (i.e. for the same Fourier frequency) by the presence of sharp power spectrum peaks. By dividing the sample spectrum by the smoothed one a white-noise like spectrum is obtained, the approximate probability distribution function $(p d f)$ of which is derived based on the characteristics of the sample spectrum. A search for coherent pulsations is then carried out by looking for peaks in the divided spectrum, for which the probability of chance occurrence is below a given detection level. If no significant peaks are found, an upper limit to the amplitude of a sinusoidal modulation is worked out for each searched frequency.

Our treatment assumes that the instrumental noise is due to Poisson statistics; as such it can be readily applied to observations with photon counting detectors in any band of the electromagnetic spectrum. The generalisation to the case of a Gaussian instrumental noise is straightforward. The time series are supposed to be equispaced and continuous.

The paper is structured as follows: in section 2 we introduce a simple approximation to the $p d f$ of the power estimates in the sample spectrum of cosmic sources characterised by "coloured" noise. Section 3 describes the smoothing algorithm that we devised in order to estimate the corresponding continuum power spectrum components, even in the presence of quite steep red-noises. In section 4 we derive the $p d f$ of the white-noise like spectrum that is obtained by dividing the sample spectrum by the smoothed one. The prescription for detecting significant periodic signals and deriving their amplitude, is given in section 5. This includes also how to obtain upper limits in the case in which no significant peak is found. Section 3-5 summarise also the results from the extensive numerical simulations that were carried out in order to assess the reliability of the technique. In section 6 an application to the "coloured" power spectra from the 
$\mathrm{X}$-ray light curves of an accreting $\mathrm{X}$-ray pulsar (V0332+53) and a bright galactic bulge source $(\mathrm{GX} 13+1)$ is presented. Our conclusions are in section 7 .

\section{THE DISTRIBUTION OF THE POWER ESTIMATES IN THE SAMPLE SPECTRUM}

When "coloured" continuum power spectrum components resulting from source variability are present, the statistical distribution of the corresponding power estimates cannot be derived in general from first principles. In the presence of extensive and repeated observations, the statistical properties of these components could be obtained directly from the data. In practice this is difficult to do, because of the limited duration of the observation and the characteristic red-noise spectra that are commonly found. An additional limitation derives from the fact that many cosmic sources display different activity states, often characterised by different luminosity and/or energy spectrum properties. A given activity state can last for time intervals as short as minutes; in same cases this imposes the tightest constraint on the lowest frequencies that can be studied in the sample spectrum, without violating the hypothesis of stationarity.

A single sample spectrum is often calculated over the entire observation duration, $T$, in order to explore the lowest possible frequencies, while maintaining the highest Fourier resolution $\left(\Delta \nu_{F}=1 / T\right)$. In this case only one power estimate is obtained for each Fourier frequency and the statistical distribution of the noise component(s) from the source remains unexplored.

Alternatively the observation can be divided in a series of $M$ consecutive intervals and the distribution of the power estimates investigated over the ensemble of the sample spectra from individual intervals of duration $T / M$. To illustrate this, we analysed a $\sim 5.5 \mathrm{hr}$ long observation of the accreting black hole candidate $\mathrm{Cyg} \mathrm{X}-1$ in its so-called "low state", one of the most variable $\mathrm{X}$-ray binary sources in the sky. The $7.8 \mathrm{~ms}$ resolved $\mathrm{X}$-ray light curve (1-20 keV energy range) was divided into $M=1244$ intervals of $16 \mathrm{~s}$ and a sample spectrum calculated for each interval. The sample spectrum obtained by averaging these $M$ spectra is given in fig. 1a. Fig. 1b shows the distribution of the power estimates for selected frequencies over the $M$ sample spectra. Each distribution is normalised by $2 /\left\langle P_{j}\right\rangle$, where $\left\langle P_{j}\right\rangle$ is the estimate of the average power at the $j$-th Fourier frequency $\nu_{j}$. It is apparent that in all cases the distribution is close to a $\chi_{2}^{2} p d f$ (also plotted for comparison). A Kolmogorov-Smirnov test gives a probability of $\sim 20-60 \%$ that the observed distributions result from a $\chi^{2} p d f$. Similar results were obtained for the sample spectra from the light curves of a few other accreting compact stars in X-ray binaries. By extrapolating these results, we assume that, (for a given activity state), the "coloured" noise components in the sample spectra of cosmic sources also follow a rescaled $\chi_{2}^{2}$-distribution ${ }^{3}$ (see also van der Klis 1988).

\footnotetext{
${ }^{3}$ Some caution is necessary for red noise spectra with a power law slope steeper than -2 . In these cases the source
} 
There is at least a very important class of random processes for which the power spectral estimates possess properties compatible with those discussed above. These are linear processes, $y(t)$, in which a white noise, $z(t)$, is passed through a linear filter $h(t)$, i.e.

$$
y(t)-\mu=\int_{0}^{\infty} h(\tau) z(t-\tau) d \tau,
$$

where $\mu=E[y(t)]$ is the mean of $y(t)$ and $E[z(t)]=0$. The power spectrum, $\Gamma_{y}$ of a linear process is given by:

$$
\Gamma_{y}(\nu)=|H(\nu)|^{2} \Gamma_{z}(\nu),
$$

where $\Gamma_{z}$ is the power spectrum of $z(t)$ and $H(\nu)$ is the frequency response function of the linear filter $h(t)$. The power spectrum is the average over the realisations of the sample spectrum, i.e. $\Gamma_{y}(\nu)=E[Y(\nu)]$ and $\Gamma_{z}(\nu)=E[Z(\nu)]=2$. The latest equality implies that the sample spectrum of the input white noise is normalised such as to follow a $\chi_{2}^{2} p d f$ (e.g. Jenkins \& Watts 1968). Given a white noise source and a suitable linear filter it is then possible to generate a random process with arbitrary spectrum. In particular, it follows from eq. 2 that for a given frequency $\nu$, the sample spectrum, $Y(\nu)$, of the linear process follows the same $\chi_{2}^{2}$ distribution of the sample spectrum of the input white noise $Z(\nu)$, except for a rescaling factor of $|H(\nu)|^{2}$. Therefore, the $p d f$ of $Y(\nu)$ is

$$
f_{\nu}(y)=\frac{e^{-y / 2|H(\nu)|^{2}}}{2|H(\nu)|^{2}}(y) .
$$

Based on the discussion above we adopt linear processes to model the sample power spectra (and their $p d f$ ) resulting from "coloured" noise variability of cosmic sources.

In practical applications the sample spectrum of astronomical time series $P_{j}$ comprises a white noise component resulting from measurement uncertainties (Poisson noise in the case of photon counting detectors). The power estimates of the white noise resulting from Poisson statistics are distributed according to a $\chi_{2}^{2} p d f$, if the normalisation

$$
P_{j}=\frac{2}{N_{\gamma}}\left|a_{j}\right|^{2}
$$

is adopted, where $N_{\gamma}$ is the total number of photons in the light curve and $a_{j}$ the complex Fourier amplitudes (see e.g. Leahy et al. 1983). In the case of a Gaussian instrumental noise with mean zero and variance $\sigma^{2}, N_{\gamma}$ is to be replaced by $N \sigma^{2}$, where $N$ is the number of points in the light curve. Therefore in the regions of the sample spectrum which are dominated by instrumental (white) noise, the power estimates will follow a $\chi_{2}^{2} p d f$. We assume that this instrumental white noise component can be interpreted as the input process $z(t)$, such that eq. (1)

variability on timescales comparable to those over which the sample spectrum is calculated, can cause a substantial low-frequency leakage, which in turn might alter the distribution of the power estimates. To limit the effects of this leakage our technique includes the possibility of subtracting polynomial trends from the light curves (see Deeter 1984). 
still holds. While clearly non-physical, this assumption involves no (statistical) approximation and allows to considerably simplify our treatment. In particular it follows that if the square modulus of the frequency response function $|H(\nu)|^{2}$ were known, then multiplying eq. (2) by $|H(\nu)|^{-2}$ the spectrum of the (instrumental) white noise would be recovered. In this case the search for significant power spectrum peaks arising from a periodic signal could be carried out by using standard techniques. In practice $|H(\nu)|^{2}$ must be estimated through the sample spectrum. One possibility would be to model the power spectrum continuum components by adopting an appropriate maximum likelihood technique (Anderson, Duvall \& Jeffries 1990; Stella et al. 1996; Arlandi et al. 1996) and use the best fit function to estimate $|H(\nu)|^{2}$. This approach, however, faces difficulties with the subjective choice of the model function and, more crucially, the estimate of the statistical uncertainties of the best fit at any given frequency. Therefore we prefer to evaluate $|H(\nu)|^{2}$ through a suitable smoothing algorithm as described in sect. 3 .

The discussion above concentrates on individual sample spectra. It is not uncommon, however, that the sample spectrum is obtained from the sum of the sample spectra of $M$ different intervals. In general, therefore, each spectral estimate can be the sum of $M$ individual estimates and its statistical distribution is, therefore, related to a $\chi^{2}$-distribution with $2 M$ degrees of freedom $(d o f)$. This case is described in detail in the Appendix.

\section{THE SMOOTHING ALGORITHM}

As the goal of any periodicity search is to detect a sharp peak over the underlying sample spectrum continuum, the power in a (possible) peak should not affect the estimate of the continuum (otherwise the sensitivity of the search would be reduced). This implies that for each frequency $\nu_{j}$ the continuum should be estimated through an interpolation of the sample spectrum which excludes $P_{j}$ itself and uses the power estimates over a range of nearby frequencies at the left and right of $\nu_{j}$. In the language of the smoothing functions, this corresponds to a well-known class of spectral windows which are zero-valued at the central frequency. We adopt for semplicity a rectangular window (with a central gap) that extends over a total of $I$ Fourier frequencies, giving a width of $\Delta \nu_{t o t}=I \Delta \nu_{F}$.

In conventional smoothing $(I-1) / 2$ Fourier frequencies are used shortwards and longwards of the central frequency $\nu_{j}$, such that the same smoothing width $\Delta \nu_{\text {left }}=\Delta \nu_{\text {right }}=(I-1) \Delta \nu_{F} / 2$ is obtained on both sides of the central frequency. The problem with this kind of smoothing is that it does not approximate with acceptable accuracy the steep power-law like red-noise components that are often found in the sample spectra of cosmic sources. Fig. 2 shows the results of 100 simulations of three different types of red power spectra consisting of: a Lorentzian centered at zero frequency (spectrum A), a power-law with a slope of -1.5 (spectrum B) and a power law with a slope of -2 (spectrum $\mathrm{C}$ ). In all cases a quasi-periodic oscillation broad peak centered around 
$100 \mathrm{~Hz}$ was included, together with a counting statistics white noise component. A smoothing width of $I=30$ Fourier frequencies was used. It is apparent that while conventional smoothing (dotted lines in fig. 2) reproduces fairly accurately the characteristics of spectrum A, it fails to reproduce the steep decay from the lowest frequencies of spectrum B and C. Moreover edge effects dominate the estimate of the smoothed spectrum for the first $(I-1) / 2$ frequencies.

A far better result is obtained if the smoothing over $I$ Fourier frequencies is distributed such that its logarithmic frequency width is (approximately) the same on both sides of $\nu_{j}$, i.e. $\log \left(\Delta \nu_{j, \text { left }}\right)=\log \left(\Delta \nu_{j, \text { right }}\right)$. This approach builds on the obvious fact that a power law spectrum is a straight line in a log-log representation. Considering that $\Delta \nu_{t o t}=\Delta \nu_{j, l e f t}+\Delta \nu_{j, r i g h t}$ it follows that

$$
\left\{\begin{array}{l}
\Delta \nu_{j, \text { left }}=\Delta \nu_{\text {tot }}-\Delta \nu_{j, \text { right }} \\
\Delta \nu_{j, \text { right }}=\frac{\left[-\left(2 \nu_{j}-\Delta \nu_{t o t}\right)+\left(4 \nu_{j}^{2}+\Delta \nu_{t o t}^{2}\right)^{1 / 2}\right]}{2}
\end{array}\right.
$$

In this scheme the smoothed spectrum $S_{j}(I)$, that we adopt as the estimator of $2\left|H\left(\nu_{j}\right)\right|^{2}$, is calculated as follows

$$
S_{j}(I)=\frac{1}{2 I_{j, l e f t}} \sum_{i=j-I_{j, l e f t}}^{j-1} P_{i}+\frac{1}{2 I_{j, r i g h t}} \sum_{i=j+1}^{j+I_{j, r i g h t}} P_{i}
$$

where $I_{j, l e f t}$ and $I_{j, r i g h t}$ (rounded to the nearest integers) are the number of Fourier frequencies in $\Delta \nu_{j, l e f t}$ and $\Delta \nu_{j, r i g h t}$, respectively.

$$
\sigma_{S_{j}(I)}^{2}=\frac{1}{2} \sqrt{\left[\frac{1}{I_{j, l e f t}} \sum_{i=j-I_{j, l e f t}}^{j-1} P_{i}\right]^{2}+\left[\frac{1}{I_{j, \text { right }}} \sum_{i=j+1}^{j+I_{j, \text { right }}} P_{i}\right]^{2}} .
$$

By propagating eq. 0 we obtain the variance $2 P_{i}$ of the $P_{i}$ variables over the smoothing formula (cf. eq. 6) The solid lines in fig. 2 show the estimate of the continuum power spectrum components (and therefore of $2\left|H\left(\nu_{j}\right)\right|^{2}$ ) obtained by using the above technique; it is apparent that also the low-frequency end of spectra B and $\mathrm{C}$ is reproduced quite well, and that edge effects are nearly absent.

In general $I$, the number of Fourier frequencies defining the smoothing width is to be adjusted so as to closely follow the sharpest continuous features of the sample spectrum (samething that favors low values of $I$ ), while mantaining the noise of the smoothed spectrum as low as possible (something that favors high values of $I$ ). To this aim we consider the divided sample spectrum $R_{j}(I)=2 P_{j} / S_{j}(I)$, i.e. the ratio of the sample spectrum and the smoothed spectrum for a range of different values of $I$. If $S_{j}(I) / 2$ provides a reasonably good estimate of $\left|H\left(\nu_{j}\right)\right|^{2}$, then $R_{j}(I)$ will approximately follow the $\chi_{2}^{2}$-distribution of the input white noise, at least for relatively small values of $R_{j}(I)$ ( $\leq 15-20$, see sect. 4). A Kolmogorov-Smirnov (KS) test can be used in order to derive out of different trial values of the width $I$, the one that makes the distribution of $R_{j}(I)$ closest to a $\chi_{2}^{2} p d f$. The KS test is especially sensitive to differences away from the tails of the distributions (see e.g. Press et al. 1992). 
Fig. 3 shows the results from simulations in which the KS probability is calculated as a function of $I$ for four different types of spectra each with 5000 Fourier frequencies. Each point in fig. 3 represents the average over 100 simulations. The second, third and fourth panels refer to spectra A, B and C of fig. 2, respectively. In all three cases the KS probability shows a broad maximum around values of $I \sim 100$. For higher values of $I$ the smoothed spectrum becomes gradually less accurate in reproducing the shape of the sample spectrum, whereas for lower values of $I$ the scatter in the estimates of $S_{j}(I)$ plays an increasingly important role in distorting the $p d f$ of $R_{j}(I)$ away from a $\chi_{2}^{2}$-distribution. Note that, as expected, the KS probability monotonically increases with $I$ in the case of a white noise sample spectrum (see upper panel of fig. 3 ).

In the following we adopt $S_{j}\left(I_{o}\right) / 2$, the smoothed sample spectrum with a width $I_{o}$ that maximises the probability of the KS test described above. In practice values of $I_{o}$ between 30-40 and the number of Fourier frequencies in the sample spectrum are to be used (see sect. 4).

\section{THE DISTRIBUTION OF THE DIVIDED SAMPLE SPECTRUM $R_{j}(I)$}

The smoothed sample spectrum $S_{j}\left(I_{o}\right) / 2$ provides our estimate of $\left|H\left(\nu_{j}\right)\right|^{2}$, in the sense discussed in the previous section. Therefore we adopt the divided spectrum $R_{j}\left(I_{o}\right)=2 P_{j} / S_{j}\left(I_{o}\right)$ as the estimator of the white noise spectrum of the input linear process. The search for coherent periodicities in the data thus translates into the problem of detecting significant peaks in $R_{j}\left(I_{o}\right)$. This, in turn, requires a detailed knowledge of the expected $p d f$ of $R_{j}\left(I_{o}\right)$, especially for high values.

For each Fourier frequency $\nu_{j}, R_{j}\left(I_{o}\right)$ is to be regarded as the ratio of the random variables $P_{j}$ and $S_{j}\left(I_{o}\right) . P_{j}$ is distributed like a $\chi_{2}^{2} p d f$ rescaled to an expectation value of $\left|H\left(\nu_{j}\right)\right|^{2}$. By approximating $\left|H\left(\nu_{j}\right)\right|^{2}$ with $S_{j}\left(I_{o}\right) / 2$ we have:

$$
f_{P_{j}}(p)=\frac{e^{-p / S_{j}\left(I_{o}\right)}}{S_{j}\left(I_{o}\right)}(p)
$$

The distribution of $S_{j}\left(I_{o}\right)$ is in general a suitable linear combination of the $I_{o}-1$ random variables $P_{j}$ used in the smoothing. These, in turn, are distributed like a rescaled $\chi_{2}^{2}$ (cf. eq. 8). For sufficiently high values of $I_{o}$, one can appeal to the central limit theorem and approximate the distribution of $S_{j}\left(I_{o}\right)$ with a Gaussian distribution of mean $S_{j}\left(I_{o}\right)$ and variance $\sigma_{S_{j}\left(I_{o}\right)}^{2}$ (cf. eq. 6 and (7), i.e.

$$
f_{S_{j}\left(I_{o}\right)}(s)=\frac{1}{\sigma_{S_{j}\left(I_{o}\right)}^{2} \sqrt{2 \pi}} e^{\left[-\left(s-S_{j}\left(I_{o}\right)\right)^{2} / 2 \sigma_{S_{j}\left(I_{o}\right)}^{2}\right]} .
$$

Note that $P_{j}$ and $S_{j}\left(I_{o}\right)$ can be regarded, for any given $j$, as statistically independent variables (indeed $P_{j}$ is not used in the computation of $S_{j}\left(I_{o}\right)$ ). In this case the $p d f$ of $R_{j}\left(I_{o}\right)$ can be written 
as (e.g. Mood, Graybill \& Boes 1974)

$$
\begin{aligned}
f_{R_{j}\left(I_{o}\right)}(r) & =\int_{-\infty}^{+\infty}|s| f_{P_{j}, S_{j}\left(I_{o}\right)}(r s, s) d s \\
& =\frac{1}{2 S_{j}\left(I_{o}\right) \sigma_{S_{j}\left(I_{o}\right)} \sqrt{2 \pi}} \cdot \int_{0}^{+\infty} s \exp \left[-\frac{(s-\mu)^{2}}{2 \sigma_{S_{j}\left(I_{o}\right)}^{2}}-\frac{r s}{S_{j}\left(I_{o}\right)}\right] d s
\end{aligned}
$$

where we have used the fact that the joint $p d f f_{\left.P_{j}, S_{j}\left(I_{o}\right)\right)}(p, s)$ is given by the product of $f_{P_{j}}(p)$ and $f_{S_{j}\left(I_{o}\right)}(s)$.

To check the accuracy and range of applicability of the $p d f$ in eq. 10, we carried out extensive numerical simulations. Figure 4 shows the results from $2 \times 10^{4}$ simulations of white noise sample spectra each cointaing 5000 Fourier frequencies (i.e. a statistics of $10^{8}$ points). The observed distribution of the $R_{j}\left(I_{o}\right)$ is shown together with the expected $p d f$ derived above. (The $p d f$ in eq. 10 and its cumulative distribution were evaluated numerically through Gaussian integration routines). In order to the avoid large values of $\sigma_{S_{j}\left(I_{o}\right)}$ arising from small values of $I_{\text {left }}$ or $I_{\text {right }}$, respectively close to the low-frequency and the high-frequency end of the sample spectrum (see eq. 母), only the powers corresponding to the Fourier frequencies from $\mathrm{j}=6$ to $\mathrm{j}=4995$ were considered. The simulations were repeated for different choices of the smoothing width I. It is apparent that while in the cases $I=50$ and 40 the $p d f$ in eq. 10 provides a very good approximation of the observed distribution, for $I=30$ and, especially, $I=20$ the expected $p d f$ shows a significant excess for values of $R_{j}(I)$ larger than $20-30$. This effect is due to the fact that the low-value end of the Gaussian approximation for the $p d f$ of $S_{j}(I)$ becomes increasingly inaccurate as $I$ decreases. Therefore in most practical application it is best to use $I \geq 40$. On the other hand, being in excess of the observed distribution, the $p d f$ in eq. 10 would artificially decrease the sensitivity of searches for significant power spectrum peaks when used with $I \simeq 20-30$, but would not favor the detection of spurious peaks.

We also tested the reliability of our approximations for the first few Fourier frequencies of the power spectra (where $I_{\text {left }} \ll I_{\text {right }}$ ) and the frequencies close to the Nyquist frequency (where $\left.I_{\text {left }} \gg I_{\text {right }}\right)$. To this aim we carried out $10^{6}$ simulations of 1000 Fourier frequencies power spectra from a white noise process, and concentrated on the distribution of $R_{j}\left(I_{o}\right)$ for $I_{o}=100$ and selected values of $j$. Fig. 5 and 6 shows a comparison of the sample and expected distributions for $j=5,6,7$ and 10 and for $j=990,993,994$ and 995 . The results clearly show that, for the $p d f$ in eq. 10 to provide a good approximation to the simulated distributions it is necessary to exclude the first and the last $5-6$ frequencies of the power spectra. The results above were also determined to be insensitive to the value of $I_{o}$, as long as $I_{o} \geq 40$.

Finally $2 \times 10^{4}$ simulations of 5000 Fourier frequencies sample spectra were carried out for the red noise spectrum B of sect. 3. The simulated and predicted $p d f$ of $R_{j}(I)$ are shown in fig. 7 for $I=30,100,200$ and 500 . The values of $R_{j}(I)$ corresponding to the first and last five Fourier frequencies were excluded from the distributions. Unlike the white noise simulations, the width $I$ is here crucial in determining whether or not the smoothed spectrum closely follows the continuum 
features of the sample spectrum. It is seen that the expected $p d f$ closely follows the simulated distribution for $I=30$ and $I=100$, whereas for $I=200$ and, especially, $I=500$ the occurence of high values of $R_{j}(I)$ is systematically in excess of the expected $p d f$. The latter effect is clearly due to the fact that, for high values of $I, S_{j}(I)$ is "too smooth" given the characteristics of the red spectrum. Note that $I=100$ is close to $I_{o}$, i.e. the value that maximises the Kolmogorov-Smirnov probability in the simulations of sect. 3 for red noise spectrum B.

It is clear from the discussion above that except for the first and last 5-6 Fourier frequencies of each spectrum, the approximate $p d f$ derived above for $R_{j}\left(I_{o}\right)$ provides accurate results, as long as $I_{o}>30-40$.

\section{PERIODIC SIGNAL DETECTION}

The technique described above was developed in order to approximate through a suitable smoothing the coloured noise components from the source variability and recover a white noise sample spectrum in which the search for narrow peaks can be carried out by applying the $p d f$ of the divided spectrum (sect. 4). In this section we outline the procedure for the detection of periodic signals and the derivation of upper limits in case no a periodic signal is found.

Given a sample spectrum $P_{j}$, the divided spectrum $R_{j}(I)=2 P_{j} / S_{j}(I)$ is calculated for a given smoothing width and its distribution compared to a $\chi_{2}^{2} p d f$ by using a KS test. This is repeated for smoothing widths ranging from a maximum of twice the number of Fourier frequencies in the sample spectrum to a minimum of $30-40$, with a spacing of half an octave. The smoothing width $I_{o}$ that is found to produce the highest KS probability is then adopted for the rest of the analysis. The divided spectrum $R_{j}\left(I_{o}\right)$ is then searched for significant peaks testyfing to the presence of a periodic modulation. The detection threshold is determined by the expected distribution of the divided sample spectrum $R_{j}\left(I_{o}\right)$, which is worked out based on the approximate $p d f$ of eq. 10 . Note that, unlike the case of a standard search in the presence of simple white noise, the detection threshold depends on the trial frequency (cf. eq. 3). By definition the threshold is given by the set of values $D_{j}\left(I_{o}\right)$ that will not be exceeded by chance at any of the $J_{\text {trial }}$ frequencies examined, with a confidence level $C$. The (small) probability $1-C$ that at least one of the $J_{\text {trial }}$ values of $R_{j}\left(I_{o}\right)$ exceeds the detection threshold is given by

$$
\begin{aligned}
1-C & =1-\left[1-\operatorname{Prob}_{\text {single }}\left(R_{j}\left(I_{o}\right)>D_{j}\left(I_{o}\right)\right)\right]^{J_{\text {trial }}} \\
& \simeq J_{\text {trial }} \operatorname{Prob}_{\text {single }}\left(R_{j}\left(I_{o}\right)>D_{j}\left(I_{o}\right)\right)
\end{aligned}
$$

where

$$
\operatorname{Prob}_{\text {single }}\left(R_{j}\left(I_{o}\right)>D_{j}\left(I_{o}\right)\right)=\int_{R_{j}\left(I_{o}\right)}^{+\infty} d r \int_{-\infty}^{+\infty}|s| f_{P_{j}, S_{j}\left(I_{o}\right)}(r s, s) d s
$$


is the chance probability of $R_{j}\left(I_{o}\right)$ exceeding the detection threshold $D_{j}\left(I_{o}\right)$. By solving the equation $\operatorname{Prob}_{\text {single }}\left(R_{j}\left(I_{o}\right)>D_{j}\left(I_{o}\right)\right) \simeq(1-C) / J_{\text {trial }}$ for $D_{j}\left(I_{o}\right)$, the detection threshold is obtained for each $j$.

The reliability of the whole procedure was tested by carrying out 1000 simulations in which 1024 frequency sample spectra with selected noise components were searched for significant peaks with a confidence level of $C=99 \%$ in each sample spectrum (the search excluded the first and last five Fourier frequencies). This was repeated for four different types of sample spectra obtained from autoregressive processes: a white noise, a red noise, a white noise plus a broad peak and a red noise plus a broad peak. Fig. 8 gives an example of each of these spectra, together with the frequencies and chance probabilities (for $J_{\text {trial }}=1014$ ) of the peaks exceeding the detection threshold. Respectively $6,11,15$ and 13 peaks above the threshold were found, consistent with the expected value of 10 .

Once a peak $R_{j^{\prime}}\left(I_{o}\right)$ above the detection threshold is found, the corresponding signal power $P_{j^{\prime}, \text { sig }}$ is obtained from the prescription of Groth (1975) and Vaughan et al. (1994) (see below). The sinusoidal amplitude, $A$, is defined by assuming that the signal is given by $C_{o}[1+A \sin (2 \pi$ $\left.\nu_{j^{\prime}, \text { sig }} t+\phi\right)$ ], with $C_{o}$ the average count rate. $A$ is then derived by using standard methods; for binned data we have (see e.g. Leahy et al. 1983).

$$
A=\left\{\left[\frac{P_{j^{\prime}, s i g}}{2}\right] \frac{4}{0.773 N_{\gamma}} \frac{(\pi j / N)^{2}}{\sin ^{2}(\pi j / N)}\right\}^{\frac{1}{2}},
$$

where $N$ is the number of bins in the light curve.

In the absence of a positive detection, the threshold $D_{j}$ must be converted to an upper limit on the (sinusoidal) signal amplitude. To this aim it is first necessary to calculate the sensitivity of the search, i.e. the weakest signal $R_{j \text {,sens }}\left(I_{o}\right)$ that will produce, with confidence $C$, a value of $R_{j}\left(I_{o}\right)$ exceeding the detection threshold $D_{j}\left(I_{o}\right)$. This is done by using the prescription of Groth (1975) and Vaughan et al. (1994) to calculate the probability distribution of the total power resulting from the signal and the (white) noise. Their procedure is adapted to our case by using $D_{j}\left(I_{o}\right)$ in place of their detection threshold for the power. Strictly speaking the $p d f$ of the noise in the divided spectrum $R_{j}\left(I_{o}\right)$ substantially exceeds the $p d f$ of a simple $\chi_{2}^{2}$ distribution for high values (see sect. 4 and fig. 4). However, the difference is only minor for values of a few (2-3) standard deviations above the average. The $p d f$ derived by Groth (1975) is therefore expected to apply to the noise in the divided spectrum, for any reasonable valuet of the confidence level $C$. $R_{j, \text { sens }}\left(I_{o}\right)$ needs to be worked out for each frequency $j$, because of the $j$-dependence of $D_{j}\left(I_{o}\right)$. For the same reason it is not possible to derive an upper limit which applies to all $j$ 's, based on the highest observed value of $R_{j}\left(I_{o}\right)$ as described by van der Klis (1988) and Vaughan et al. (1994). The set of $R_{j, s e n s}\left(I_{o}\right)$ obtained in this way are then converted to the corresponding powers

\footnotetext{
${ }^{4}$ This is unlike the derivation of the detection threshold $D_{j}\left(I_{o}\right)$ which requires a detailed knowledge of the $p d f$ for higher values, given the usually high number of $J_{\text {trial }}$.
} 
through $P_{j, \text { sens }}\left(I_{o}\right)=R_{j, \text { sens }}\left(I_{o}\right)\left(S_{j}\left(I_{o}\right) / 2\right)$ and then to the sinusoidal signal amplitude $A_{j, \text { sens }}$ by using eq. 13. A similar procedure is used to derive confidence intervals on the power $P_{j^{\prime}, \text { sig }}$, and therefore the amplitude $A$, of a detected signal ( $P_{j^{\prime}, s i g}$ is used in place of $D_{j}$ in this case).

\section{APPLICATION AND RESULTS}

In this section we apply the technique described above to the light curves of two highly variable $\mathrm{X}$-ray binaries containing an accreting neutron star. The data were obtained with the Medium Energy (ME) proportional counter array on board the EXOSAT satellite (Peacock \& White 1988).

$\mathrm{V} 0332+53$ is a $4.4 \mathrm{~s} \mathrm{X}$-ray pulsar accreting from a Be star in a transient X-ray binary. Its light curves are characterised by a pronounced red-noise type variability. The $4.4 \mathrm{~s}$ pulsations have a small amplitude ( $A \simeq 8 \%$; Stella et al. 1985 and references therein). We re-analysed a 4 hr long 1-9 keV light curve obtained on 1984 January 24 with the EXOSAT ME, during which the source was relatively faint (average rate of $\sim 36$ counts $\mathrm{s}^{-1}$ ). The light curve was binned to a time resolution of $938 \mathrm{~ms}$ and a single sample spectrum calculated over a 16384 point interval (see curve $a$ of fig. 9). A smoothing width of $I_{o}=1803$ Fourier frequencies was obtained through the KS test described in sect. 3. In order to save on computer time, a preliminary search for narrows peaks is carried out by using the rescaled $\chi_{2}^{2}$-like $p d f$ of $P_{j}$ in eq. 8 , and modifying accordingly the procedure described in sect. 5 (this is equivalent to ignoring the statistical uncertainties in the estimate of the smoothed spectrum $S_{j}\left(I_{o}\right)$ ). It is apparent from fig. 4 that a $\chi_{2}^{2} p d f$ is systematically lower than the $p d f$ of $R_{j}\left(I_{o}\right) p d f$ in eq. 8 for high values. Therefore any divided spectrum peak exceeding the detection threshold given by $D_{j}\left(I_{o}\right)$ will necessarily correspond to a $P_{j}$ which exceeds the preliminary detection threshold obtained from eq. 8. A narrow peak at a frequency of $0.229 \mathrm{~Hz}$ (period of $4.376 \mathrm{~s}$ ) was detected above the preliminary detection threshold for a $3 \sigma$ confidence level (see curve $b$ in fig. 9). It is apparent from fig. 9 that the peak lies upon an extended red-noise component with an approximate power law slope of -1 . The probability of chance occurrence of the corresponding peak in $R_{j}\left(I_{o}\right)$ was then worked out on the basis of the $p d f$ in eq. 10 and the prescription in sect. 5. This gave a probability of chance occurrence of $8.6 \times 10^{-5}$ for 8182 trial frequencies. Therefore the technique that we have developed would have led to the discovery of the $\sim 4.4 \mathrm{~s}$ pulsation period based only on the spectrum of fig. 9 . The pulsation amplitude was determined to be $7 \pm 1$ ( $1 \sigma$ error bars) consistent with the values quoted in the literature.

GX13+1 is a relatively poorly studied bright galactic bulge X-ray source, which displays only aperiodic variability up to frequencies of several tens of $\mathrm{Hz}$ (Garcia et al. 1988; Hasinger \& van der Klis 1989). The source is likely a binary system containing an old neutron star accreting from a low mass companion. We have reanalysed the 1985 EXOSAT ME observations of GX13+1, 
searching for a periodic modulation of the $\mathrm{X}$-ray flux in the frequency domain shortwards of $10^{-2} \mathrm{~Hz}$ where the red-noise variability is clearly dominant. For the $\sim 6 \mathrm{hr}$ observation of 1985 April 1 and the $\sim 7 \mathrm{hr}$ observation of 1985 May 2, light curves with a resolution of $2.5 \mathrm{~s}$ and $1 \mathrm{~s}$, respectively, were accumulated in different energy bands (1-4, 4-9 and 1-9 keV). The power spectra were calculated over the longest possible interval for each observation in order to extend the search to the lowest sampled frequencies. The self-consistent determination of the smoothing width provided values of $I_{o}$ between $\sim 67$ and $\sim 1422$ Fourier frequencies for different energy ranges. No significant peaks above the $95 \%$ confidence detection threshold were found in any of the power spectra. The corresponding upper limits to the amplitude of a sinusoidal modulation were worked out for each observation in the three different energy intervals. Two examples are given by curves $c$ in fig. 10. Values for selected trial periods are listed in Table 1 ; they range from $\sim 1 \%$ to $30 \%$ for periods between 200 and $10000 \mathrm{~s}$. The behaviour of these upper limits clearly reflects the shape of the red-noise component of the sample spectra, such that source variability, rather than counting statistics noise, limits the sensitivity of the period searches.

\section{CONCLUSION}

The power spectrum analysis technique that we developed for the detection of periodic signals in the presence of "coloured" noise components presents the following main advantages: $(i)$ it does not require any reduction of the Fourier frequency resolution; (ii) it can be used also in the presence of the relatively steep red noise components (power law slopes as low as -2) which are commonly found in nature; (iii) it takes into account the statistical uncertainties in the estimator of the continuum power spectrum components. Extensive numerical simulations were carried out in order to test the reliability of the technique and define the range of applicability of the adopted approximations. We found that very good results are obtained if the first and the last 5-6 Fourier frequencies of the sample spectrum are excluded from the analysis and the width of the smoothing is larger than 30-40 Fourier frequencies.

Though based on relatively simple statistics, the numerical evaluation of the peak detection threshold must be carried out separately for each Fourier frequency: this can be quite CPUintensive. A way around this limitation consists in carrying out a much faster search for potentially significant peaks by using a preliminary and simpler detection threshold. The significance of candidate peaks is then reassessed on the basis of a complete application of the technique described in this paper. Computer programs based the technique described in this paper will be made available to the community through the timing analysis package Xronos (Stella \& Angelini 1992a,b).

The authors acknowledge useful discussions with L. Angelini and A. Parmar. M. van der Klis 
provided useful comments on an earlier version of the paper. This work was partially supported through ASI grants.

\section{A. GENERALIZATION TO $M(>1)$ SPECTRA}

This appendix describes the more general case in which the sample spectrum is calculated by summing $M(>1)$ individual sample spectra each obtained from a separate light curve interval.

The normalisation of the power estimates in eq. 4 , is converted to

$$
P_{j}=\sum_{i=1}^{M} P_{j, i}=\sum_{i=1}^{M} \frac{2}{N_{\gamma, i}}\left|a_{j, i}\right|^{2}, \quad \sum_{i=1}^{M} N_{\gamma, i}=N_{\gamma}
$$

Being each of the $M$ variables $P_{j, i}$ distributed like a rescaled $\chi_{2}^{2}$, their sum $P_{j}$ will follow a $\chi^{2}$ distribution with $2 M d o f$, rescaled by the same factor. The $p d f$ of $P_{j}$ (cf. eq. 8) is therefore given by

$$
f_{P_{j}}(p)=\frac{1}{\Gamma(M)}\left(\frac{1}{2}\right)^{M} p^{M-1}\left(S_{j}\left(I_{o}\right)\right)^{-M} e^{-p / 2 S_{j}\left(I_{o}\right)}(p)
$$

The estimate of $\left|H\left(\nu_{j}\right)\right|^{2}$ is given by $S_{j}\left(I_{o}\right) / 2 M$ and consequently the divided spectrum is $R_{j}\left(I_{o}\right)=$ $2 M P_{j} / S_{j}\left(I_{o}\right)$. The approximate $p d f$ of $R_{j}\left(I_{o}\right)$ given in eq. 10 converts to

$$
\begin{aligned}
f_{R_{j}\left(I_{o}\right)}(r)= & \frac{1}{\sigma \sqrt{2 \pi}} \frac{2^{-M}}{\Gamma(M)} \cdot \int_{0}^{+\infty} s(r s)^{M-1}\left(S_{j}\left(I_{o}\right)\right)^{-M} \\
& \exp \left[-\frac{(s-\mu)^{2}}{2 \sigma^{2}}-\frac{r s}{2 S_{j}\left(I_{o}\right)}\right] d s .
\end{aligned}
$$

This approximate $p d f$ remains fairly accurate for $M I_{o} \geq 40$. Finally the sinusoidal signal amplitude (eq. 13) is given by

$$
A=\left\{\left[\frac{P_{j^{\prime}, s i g}}{2 M}\right] \frac{4}{0.773 N_{\gamma}} \frac{(\pi j / N)^{2}}{\sin ^{2}(\pi j / N)}\right\}^{\frac{1}{2}} .
$$

In order to test the reliability of the technique, we also carried out extensive numerical simulations for a variety of cases in which $M>1$. 


\section{REFERENCES}

Anderson, E. R., Duvall, T. L., \& Jeffries, S.M. 1990, ApJ, 364, 699.

Arlandi, E., Stella, L., \& Tagliaferri, G. 1996, in preparation.

Deeter, J. E. 1984, ApJ, 281, 482.

Ebisuzaki, T., Hanawo, T., \& Sugimoto, D. 1984, PASJ, 36, 551.

Garcia, M., Grindlay, J. E., Molnar, L. A., Stella, L., White, N. E., \& Seaquist, E. R. 1988, ApJ328m 552.

Grindlay, J. E. 1985, in Japan U.S. Seminar on Galactic and Extragalactic Compact X-ray Sources, ed. W. Lewin and Y. Tanaka (Tokyo:ISAS), p. 215.

Groth, E. J. 1975, ApJS, 29, 285.

Hasinger, G., \& van der Klis, M. 1989, A\&A, 225, 79.

Jenkins, G. M., \& Watts, D. G. 1968, Spectral Analysis and its applications, Holden-Day.

Kaspi, V. M., Taylor, J. H. \& Ryba, M. F. 1994, ApJ, 428, 713.

Leahy, D. A., Darbro, W., Elsner, R. F., Weisskopf, M. C., Sutherland, P. G., Kahn, S. \& Grindlay, J. E. 1983, ApJ, 266, 160.

Mood, A. M., Graybill, F. A., \& Boes, D. C., 1974, Introduction to the Theory of Statistics, McGrow-Hill Publishing Company.

Press, W. H., Teukolsky, S. A., Vetterling, W. T., \& Flannery, B. P. 1992, Numerical Recipes, Cambridge University Press

Scheid, F. 1968, Numerical Analysis, McGrow-Hill.

Stella L., \& Angelini A. 1992a, in Data Analysis in Astronomy IV, Eds. V. Di Gesù, L. Scarsi, R. Buccheri, P. Crane, M.C. Maccarone \& H.V. Zimmerman, (Plenum Press: New York), p. 59.

Stella L., \& Angelini A. 1992b, ESA TM-16 Ed. C. Barron (ESTEC-NL).

Stella, L., Arlandi, E., Tagliaferri, G., \& Israel, G. L. 1996 in Time Series Analysis in Astronomy and Meteorology, Ed. S. Rao, in press.

Stella L., Priedhorsky, W. C., \& White, N. E. 1987, ApJ, 312, L17.

Stella L., White, N. E., \& Taylor, B. G. 1985, in ESA SP-236, Recent Results on Cataclysmic Variables, p. 125.

Stella L., White, N. E., Davelaar, J., Parmar, A., Blisset, R. J., \& van der Klis, M. 1985, ApJ, $288, \mathrm{~L} 45$.

van der Klis, M. 1988, Proc. NATO Advanced Study Institute Timing Neutron Stars, Cesme, Turkey. 
van der Klis, M. 1995 , in X-ray Binaries, Eds. W.H.G. Lewin, J. van Paradijs \& E.P.J. van den Heuvel (Cambridge University Press), p.252 .

Vaughan, B. A., van der Klis, M., Wood, K. S., Norris, J. P., Hertz, P., Michelson, P. F., van Paradijs, J, Lewin, W. H. G., Mitsuda, K., \& Penninx W. 1994, ApJ, 435, 362.

White, N. E., \& Marshall, F. E. 1984, ApJ, 281, 354.

White, N. E., \& Peacock, A. 1988, Mem.S.A.It., 59, N.1-2, 7. 


\section{Figure captions}

Figure 1: (upper panel) Average sample spectrum from a 1-20 keV EXOSAT observation of the black hole candidate X-ray binary Cyg X-1. (lower panels) Distribution of the normalised spectral estimates for selected Fourier frequencies $(j=6,10,20,40,60$, see arrows in fig. 1a). The solid lines represent a $\chi_{2}^{2}$-distribution.

Figure 2: Comparison between the standard rectangular smoothing technique (dashed lines) and the logarithmic interval smoothing technique (solid lines) for the three "coloured" spectral shapes discussed in the text. Lines and points represent the average from 1000 simulations. The smoothing width is $I=30$. The difference between the two smoothing techniques at a single Fourier frequency is sketched in the central panel (the numbers indicate $I_{\text {left }}$ and $I_{\text {right }}$, i.e. the number of Fourier frequencies used at the left and the right of the nominal frequency $\nu_{j}$ ).

Figure 3: Kolmogorov-Smirnov probability as a function of the smoothing width $I$ for the comparison between a $\chi_{2}^{2}$-distribution and $R_{j}(I)$ in three different cases: a white noise spectrum (W) and the "coloured" spectra of fig. 2 (A, B and C).

Figure 4: Distribution of $R_{j}(I)$ from $2 \times 10^{4}$ simulations of a 5000 frequency white noise spectrum, for selected values of the smoothing width $I$. Note that only the power estimates between $\mathrm{j}=6$ and $\mathrm{j}=4995$ were considered. The lines give the expected $p d f$, calculated as described in the text. For comparison a $c h i_{2}^{2} p d f$ is also shown at the bottom of the figure.

Figure 5: Distribution of $R_{j}(I)$ from $10^{6}$ simulations of a 1000 frequency white noise spectrum, for $I_{o}=100$ and selected Fourier frequencies close to the low-frequency end $(j=5,6,7,10)$. The lines give the expected $p d f$, calculated as described in the text.

Figure 6: As fig. 5 but for the last frequencies of spectrum close to the Nyquist frequency $(j=995,994,993,990)$.

Figure 7: Distribution of $R_{j}(I)$ from $2 \times 10^{4}$ simulations of a 5000 frequency red noise spectrum, for selected smoothing widths $I$. Only the power estimates between $\mathrm{j}=6$ and $\mathrm{j}=4995$ were considered.

Figure 8: Left panels: examples of the sample spectra obtained from autoregressive processes and searched for coherent pulsations $\left(10^{3}\right.$ simulations). From top to bottom the power spectra comprise: a red noise, a white noise, a white noise plus a broad peak centered at $0.15 \mathrm{~Hz}$ and a red noise plus a broad peak centered at $0.25 \mathrm{~Hz}$ Right panels: probability of the peaks exceeding the 99\% confidence preliminary detection threshold (see sect. 6). The probability is calculated from 
the $p d f$ of $R_{j}\left(I_{o}\right)$ in eq. 10. From top to bottom only 611,15 and 13 peaks, respectively, were found to exceed to $99 \%$ confidence threshold (shown by the horizontal line). This is consistent with the expected value of 10 . Note that in the presence of red noise most of the peaks detected with the preliminary threshold turn out not be be significant.

Figure 9: Power spectrum of the 1-9 keV EXOSAT ME light curve of X-ray transient source V0332+53 obtained during the 1984 January 24 observation (a), together with the preliminary threshold for the detection of sinusoidal periodicities at $3 \sigma$ confidence level $(\mathrm{b})$. The first and last five frequencies of the power spectrum are not included in the search for periodicities.

Figure 10: Power spectra of the 1-9 keV EXOSAT ME light curves of GX13 +1 for the 1985 April 1(upper panel) and May 2(lower panel) observations (a), together with the preliminary threshold for the detection of sinusoidal periodicities at the $95 \%$ confidence level (b). Curve (c) shows the corresponding upper limits on the pulsation amplitude $A$. 
TABLE 1

GX13+1

\begin{tabular}{lcccccc}
\hline \hline \multirow{2}{*}{$\begin{array}{l}\text { Period } \\
s\end{array}$} & \multicolumn{4}{c}{$95 \%$ confidence upper limits on A } \\
\cline { 2 - 7 } & $1-9$ & $1-4$ & $4-9$ & $1-9$ & $1-4$ & $4-9$ \\
\hline 10240 & $6.3 \%$ & $11.1 \%$ & $3.7 \%$ & $20.1 \%$ & $29.6 \%$ & $12.4 \%$ \\
6830 & 5.6 & 9.1 & 6.2 & 14.5 & 21.1 & 9.2 \\
5120 & 6.2 & 8.6 & 5.9 & 10.0 & 14.7 & 6.3 \\
4096 & 4.7 & 6.5 & 6.0 & 8.3 & 12.0 & 5.4 \\
2048 & 2.4 & 3.3 & 3.0 & 4.7 & 6.9 & 2.8 \\
1024 & 1.4 & 2.3 & 1.8 & 2.5 & 3.7 & 1.7 \\
256 & 0.8 & 1.3 & 0.9 & 1.1 & 1.4 & 1.0 \\
128 & 0.7 & 0.9 & 0.8 & 0.7 & 0.9 & 0.8 \\
\hline
\end{tabular}


Cygnus $\mathrm{X}-1$
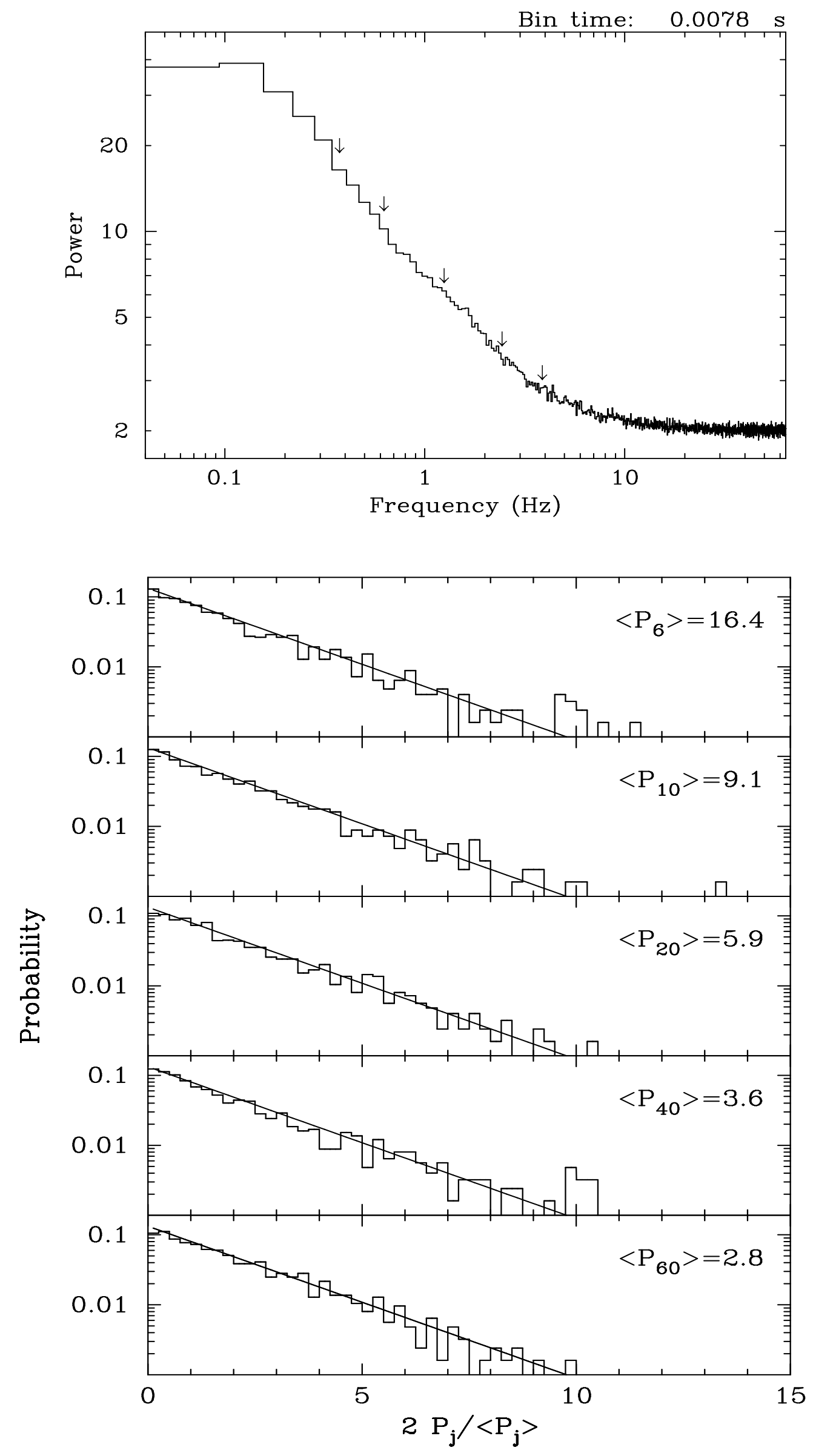

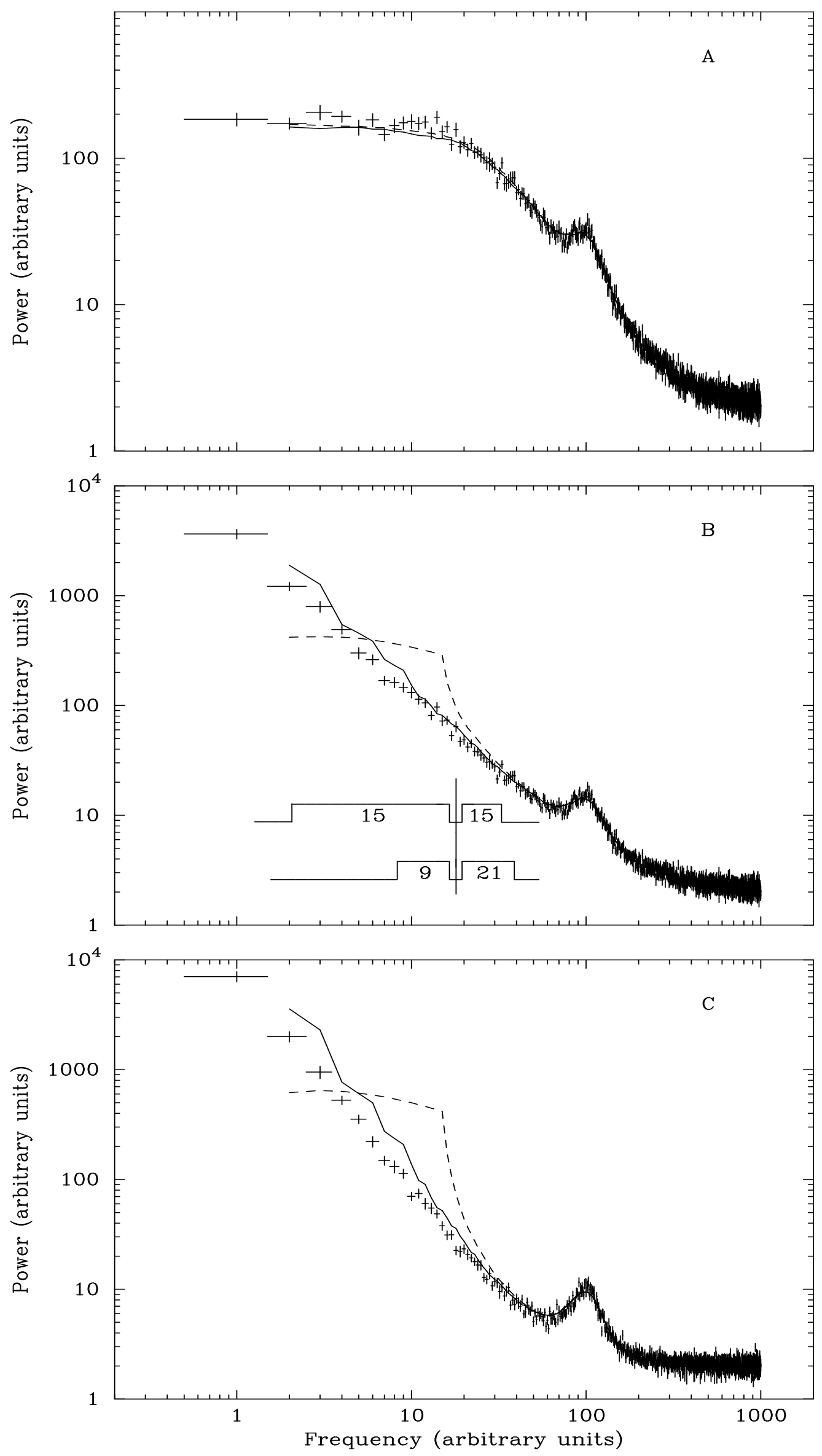


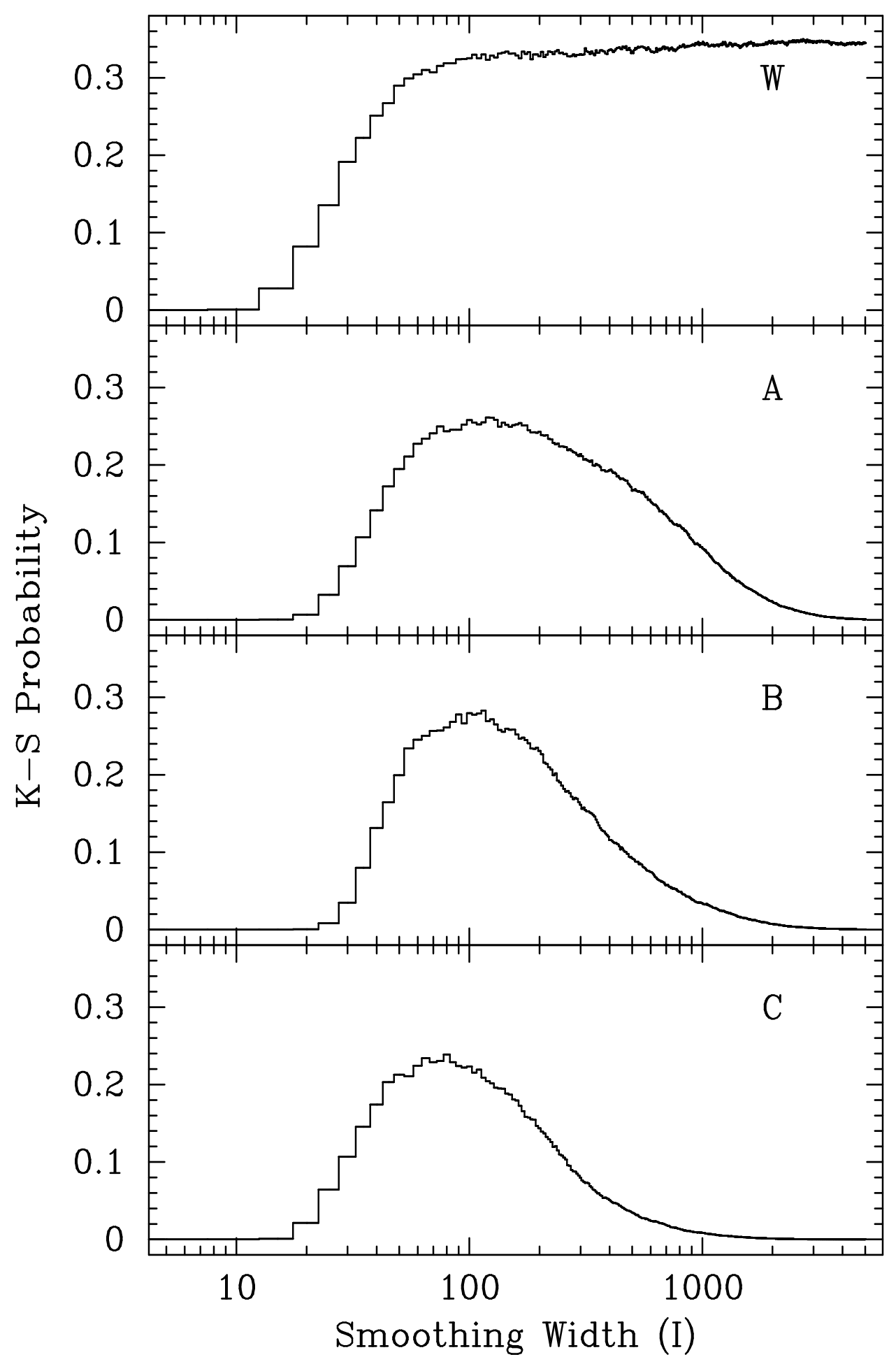




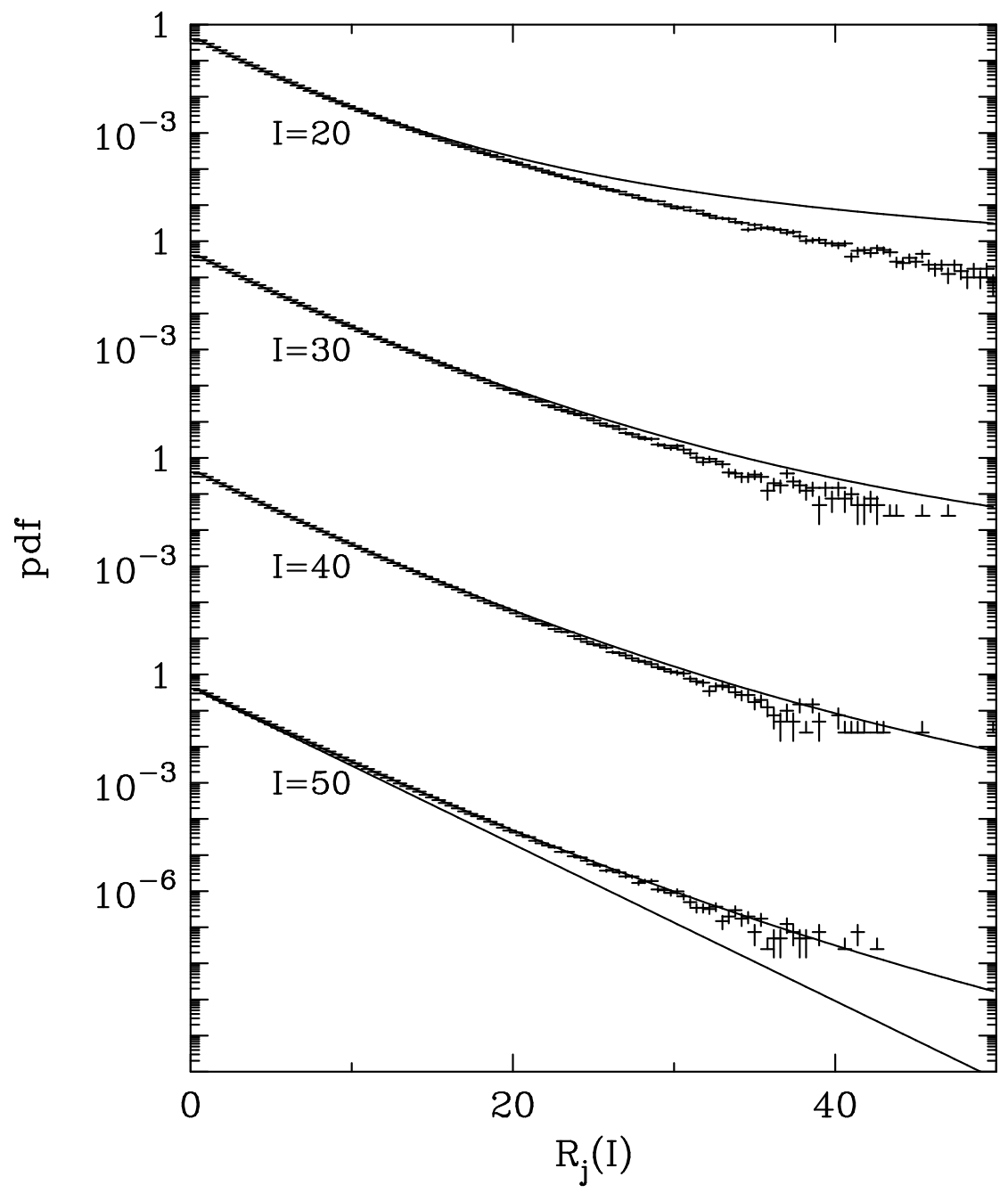




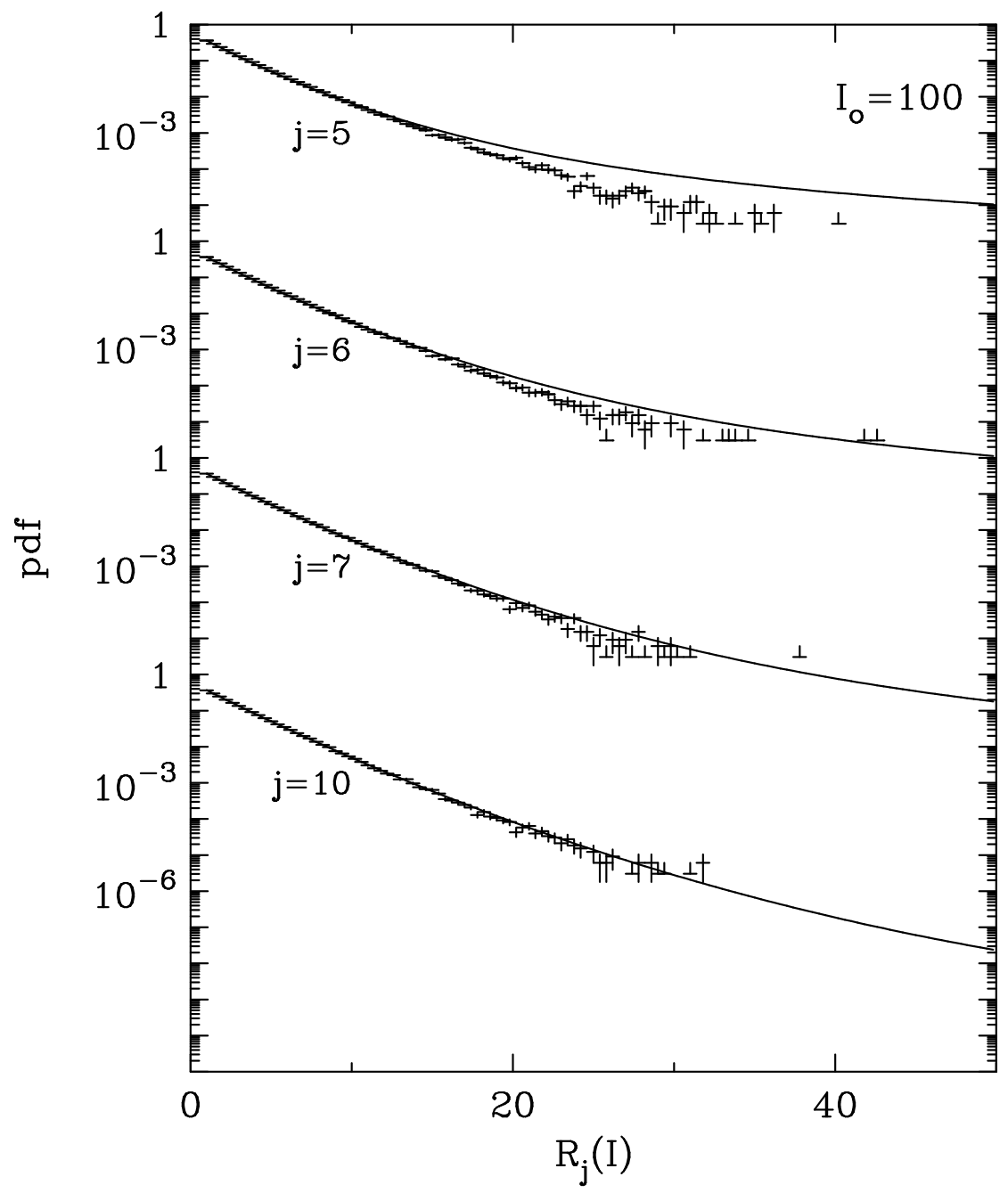




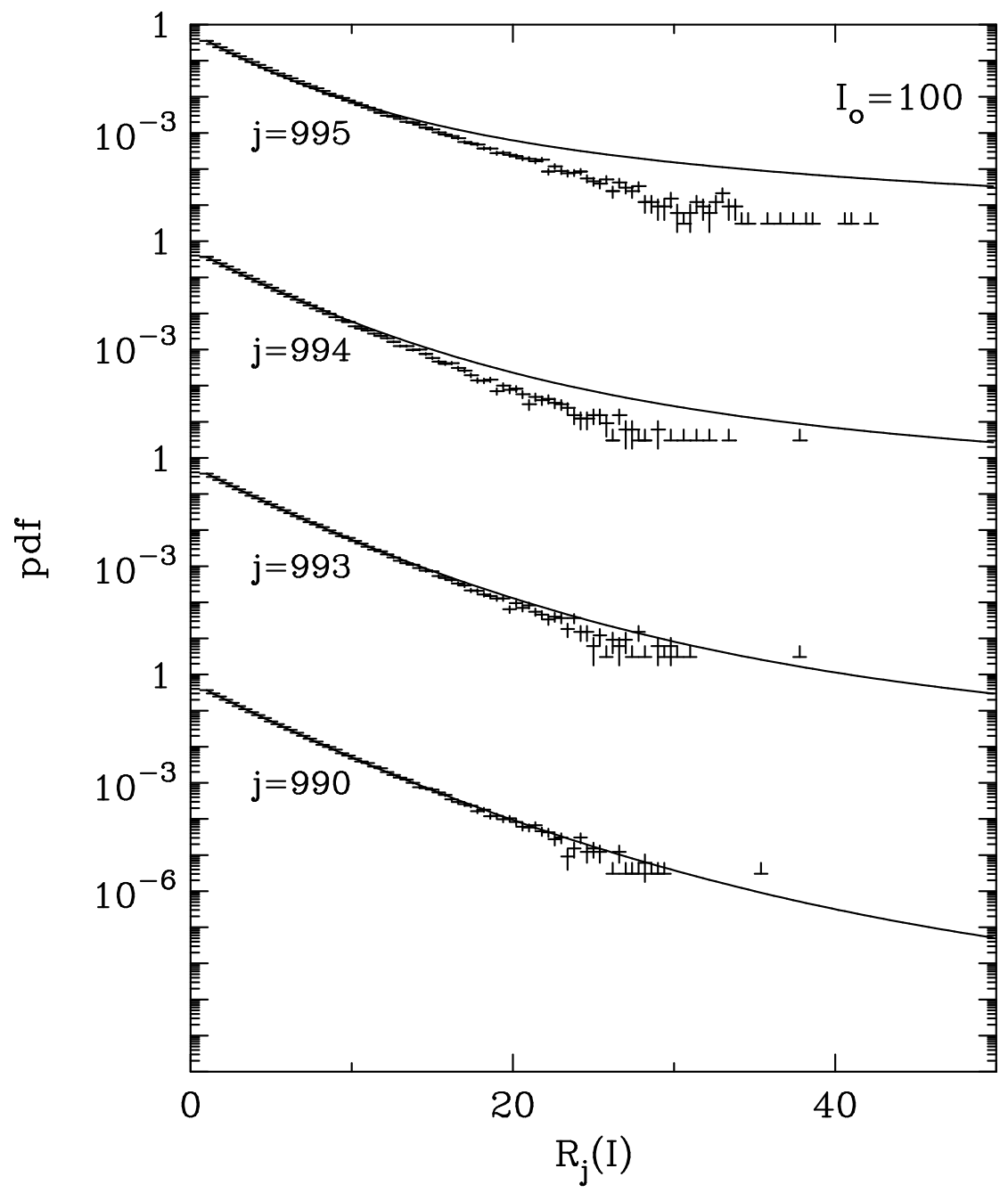




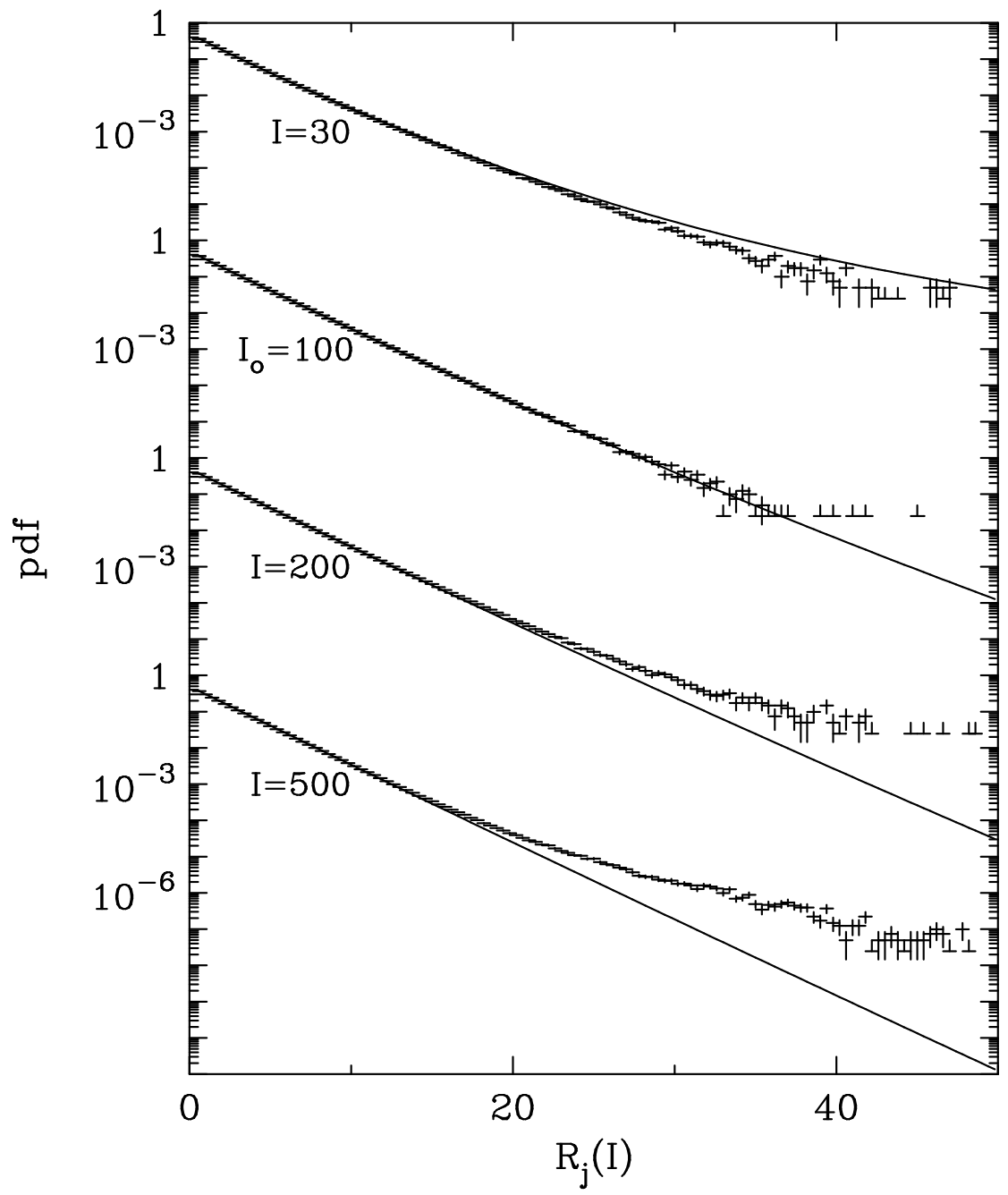



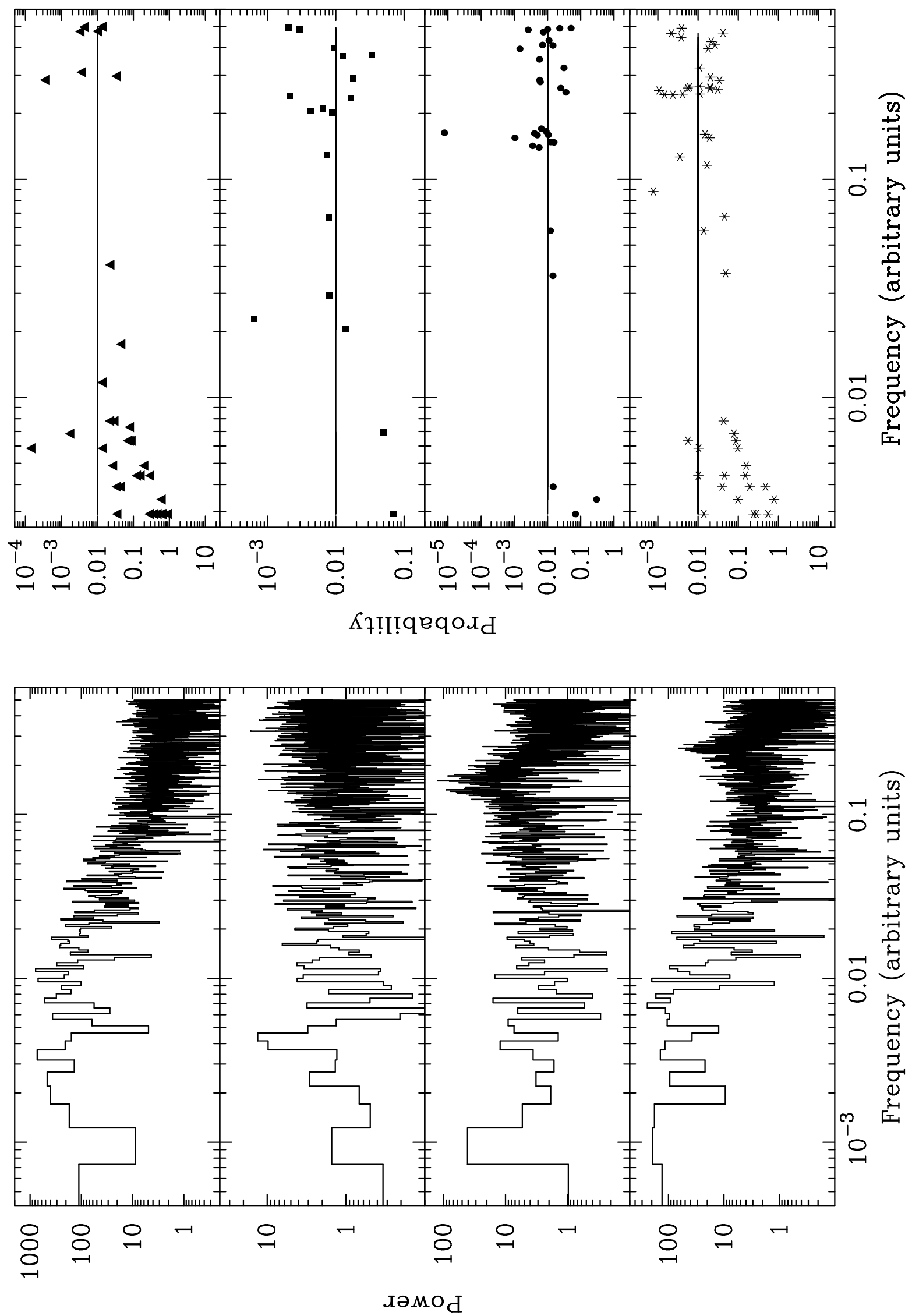


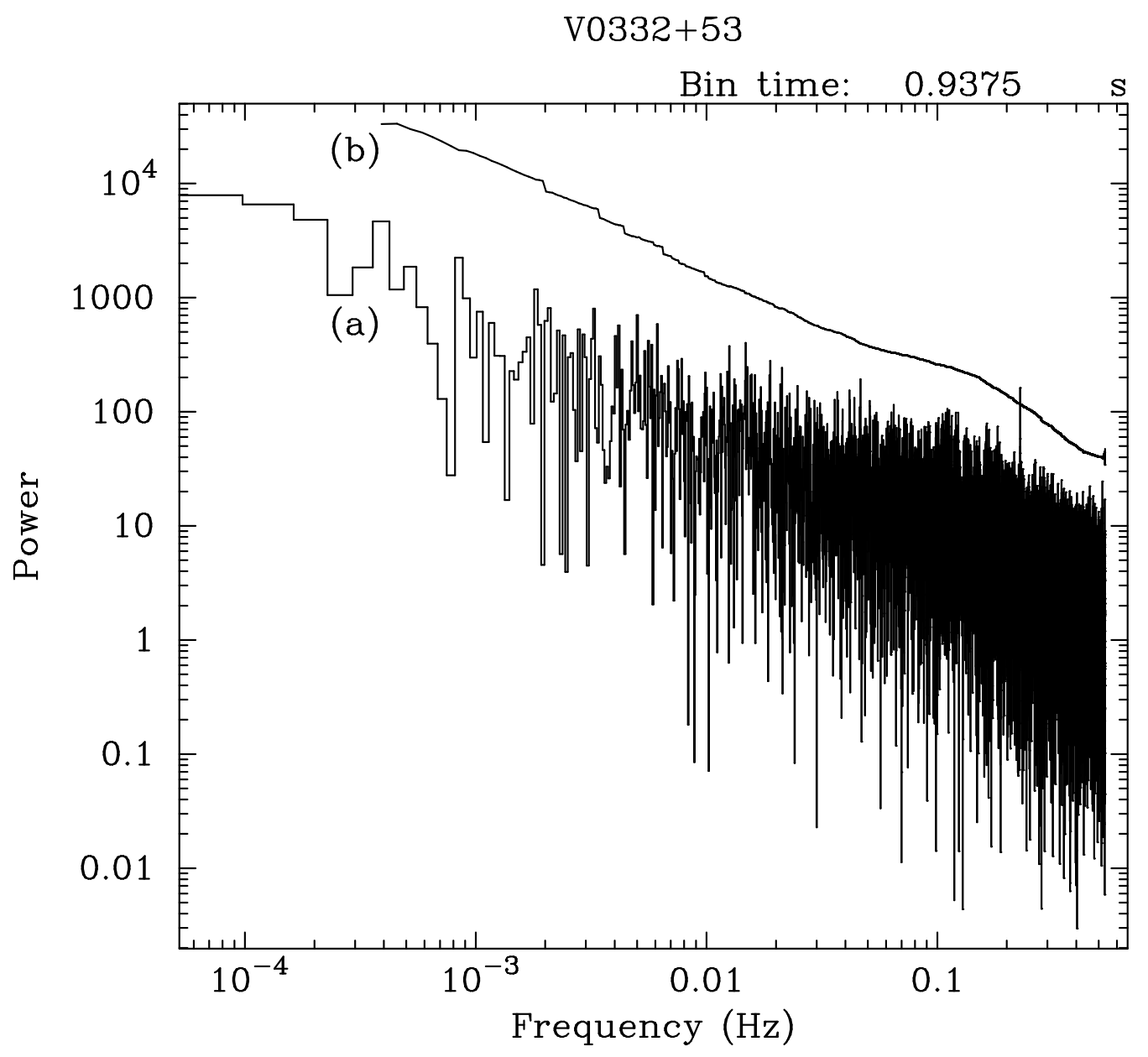



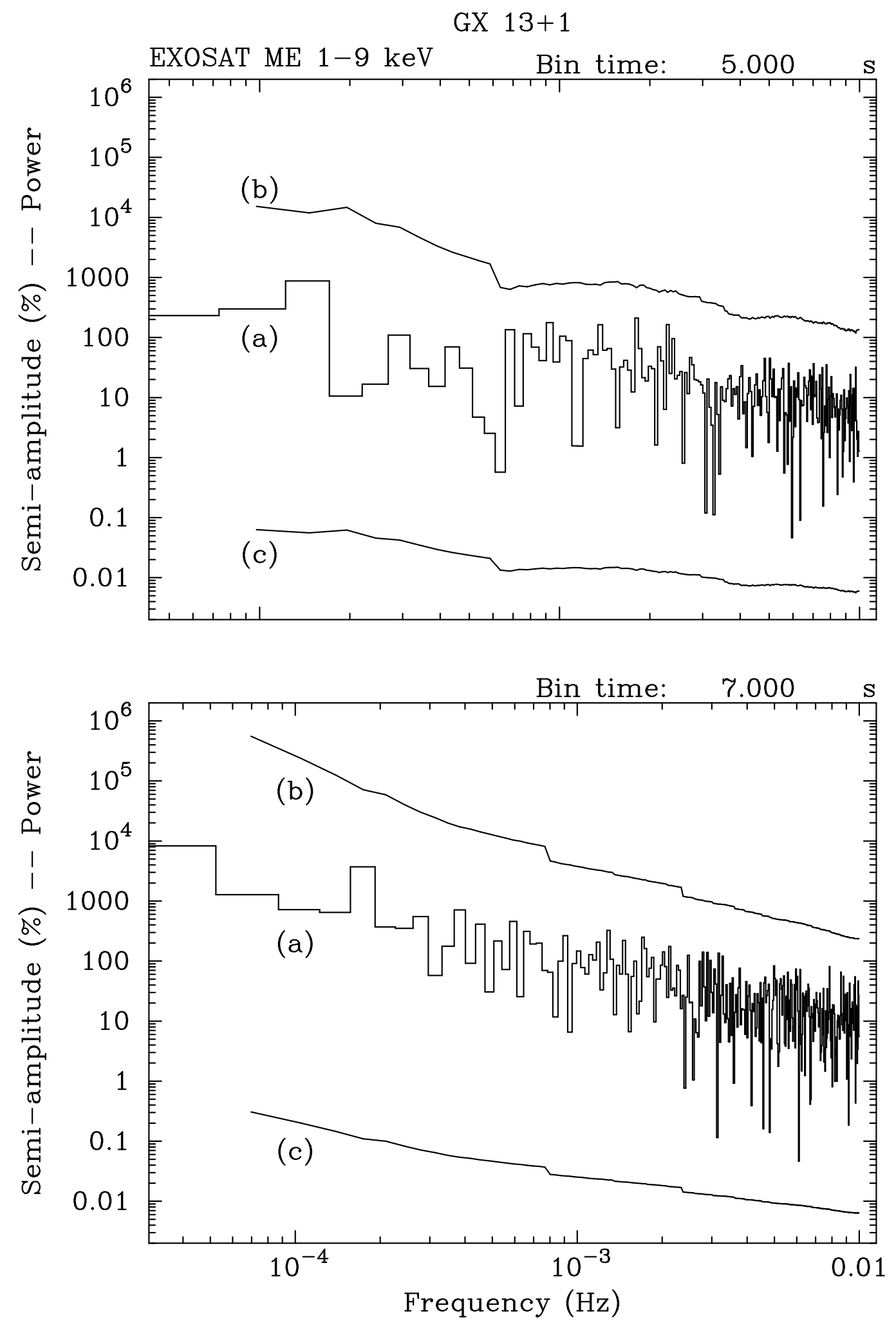